\title{
Sound and Decidable Type Inference for Functional Dependencies
}

\author{
Gregory J. Duck ${ }^{1}$, Simon Peyton-Jones ${ }^{2}$, Peter J. Stuckey ${ }^{1}$, and \\ Martin Sulzmann ${ }^{3}$ \\ 1 Department of Computer Science and Software Engineering \\ The University of Melbourne, Vic. 3010, Australia \\ $\{$ gjd,pjs\}@cs.mu.oz.au \\ 2 Microsoft Research Ltd \\ 7 JJ Thomson Avenue, Cambridge CB3 0FB, England \\ simonpj@microsoft.com \\ 3 School of Computing, National University of Singapore \\ S16 Level 5, 3 Science Drive 2, Singapore 117543 \\ sulzmann@comp.nus.edu.sg
}

\begin{abstract}
Functional dependencies are a popular and useful extension to Haskell style type classes. In this paper, we give a reformulation of functional dependencies in terms of Constraint Handling Rules (CHRs). In previous work, CHRs have been employed for describing user-programmable type extensions in the context of Haskell style type classes. Here, we make use of CHRs to provide for the first time a concise result that under some sufficient conditions, functional dependencies allow for sound and decidable type inference. The sufficient conditions imposed on functional dependencies can be very limiting. We show how to safely relax these conditions.
\end{abstract}

\section{Introduction}

Functional dependencies, introduced by Mark Jones Jon00, have proved to be a very attractive extension to multi-parameter type classes in Haskell. For example, consider a class intended to describe a collection of type c containing values of type e:

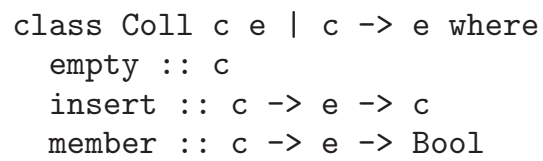

The part "| $c->e$ " is a functional dependency, and indicates that fixing the collection type $c$ should fix the element type e. These functional dependencies have proved very useful, because they allow the programmer to control the type inference process more precisely. We elaborate in Section 2

The purpose of this paper is to explore and consolidate the design space of functional dependencies (FDs). The main tool we use in this exploration is the reformulation of FDs in terms of Constraint Handling Rules (CHRs) Frü95, SS02, an idea that we review in Section 3. This reformulation allows us to make several new contributions: 
- Despite their popularity, functional dependencies have never been formalised, so far as we know. CHRs give us a language in which to explain more precisely what functional dependencies are. In particular, we are able to make the socalled "improvement rules" implied by FDs explicit in terms of CHRs.

- Based on this understanding, we provide the first concise proof that the restrictions imposed by Jones on functional dependencies [Jon00] ensure sound and decidable type inference (Section 3).

- Jones's restrictions can be very limiting. We propose several useful extensions (Section 4) such as more liberal FDs (Section 4.1). We establish some concise conditions under which liberal FDs are sound.

Throughout, we provide various examples to support the usefulness of our improvement strategies. Related work is discussed in Section 5. We conclude in Section 6]

We refer the interested reader to [DPJSS03 for proofs and additional material.

\section{Background: Functional Dependencies in Haskell}

We begin by reviewing functional dependencies, as introduced by Jones Jon00, assuming some basic familiarity with Haskell-style type classes.

Example 1. Recall the collection class

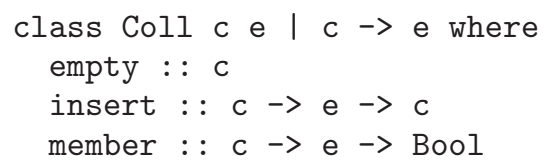

plus the following

instance Eq a $\Rightarrow$ Coll [a] a where ...

ins2 xs a b = insert (insert xs a) $b$

Consider the function ins2. In the absence of functional dependencies, type inference would give

ins2 : $(\operatorname{Coll} c$ e1, Coll c e2) $\Rightarrow c \rightarrow$ e1 $\rightarrow$ e2 $\rightarrow c$

which is of course not what we want: we expect $a$ and $b$ to have the same type. The functional dependency $c^{->e}$ expresses the idea that the collection type $c$ fixes the element type e, and hence that e1 and e2 must be the same type. In such a situation, we commonly say that types are "improved" Jon95.

Functional dependencies are useful in many different contexts. Here are some representative examples.

Example 2. Consider the following class for representing state monads and two instances 


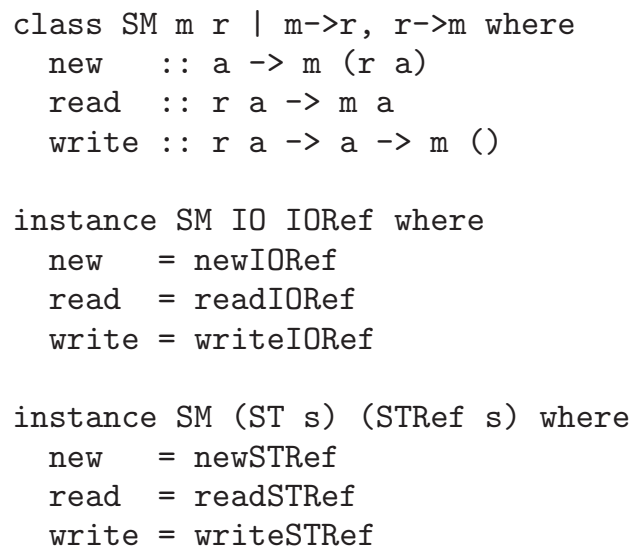

The part "| $\mathrm{m}->\mathrm{r}, \mathrm{r}->\mathrm{m}$ " gives two functional dependencies, and indicates that fixing the monad type $\mathrm{m}$ should fix the reference type $\mathrm{r}$ as well, and vice versa. Now consider the code

$$
f \mathrm{x}=\text { do }\{\mathrm{r}<- \text { new } \mathrm{x} \text {; print "Hello"; return } \mathrm{r}\}
$$

The call to print, whose type is String -> IO (), makes it clear that $f$ is in the IO monad, and hence, by the functional dependency, that $r$ must be an IORef. So we infer the type

$$
f:: a \rightarrow \text { IO (IORef a) }
$$

From this example we can see the main purpose of functional dependencies: they allow the programmer to place stronger conditions on the set of constraints generated during type inference, and thus allow more accurate types to be inferred. In their absence, we would infer the type

$$
\text { f : : (SM IO } r) \Rightarrow \text { IO ( } r \text { a) }
$$

which is needlessly general. In other situations, ambiguity would be reported. For example:

$$
\begin{aligned}
& g:: a-10 \text { a } \\
& g x=\text { do }\{r<- \text { new } x ; \operatorname{read} r\}
\end{aligned}
$$

Without functional dependencies, the type system cannot work out which reference type to use, and so reports an ambiguous use of new and read.

Example 3. Consider the following application allowing for (overloaded) multiplication among base types such as Int and Float and user-definable types such as vectors. For simplicity, we omit the obvious function bodies.

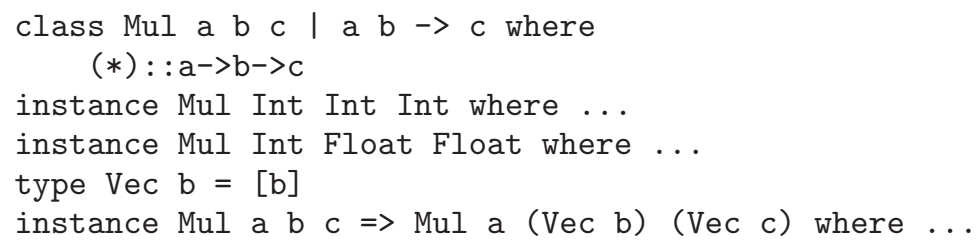


The point here is that the argument types of $(*)$ determine its result type. In the absence of this knowledge an expression such as $(a * b) * c$ cannot be typed, because the type of the intermediate result, $(a * b)$, is not determined. The type checker would report type ambiguity, just as it does when faced with the classic example of ambiguity, (read (show $\mathrm{x}$ )).

Example 4. Here is an another useful application of FDs to encode a family of zip functions.

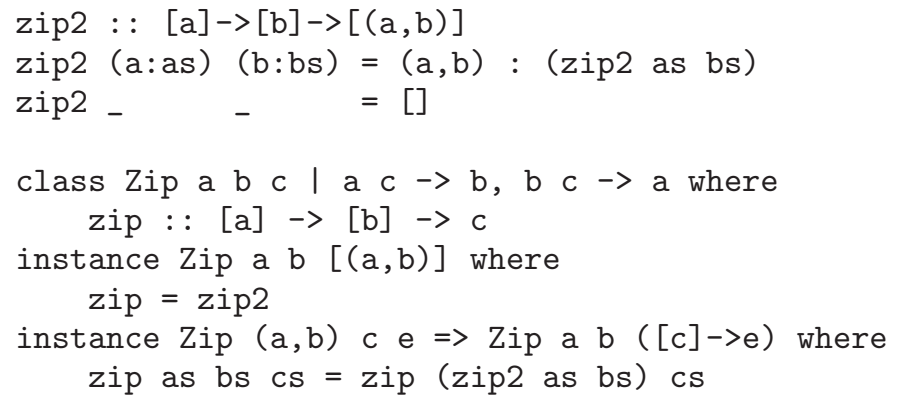

These definitions make zip into an n-ary function. For example, we may write

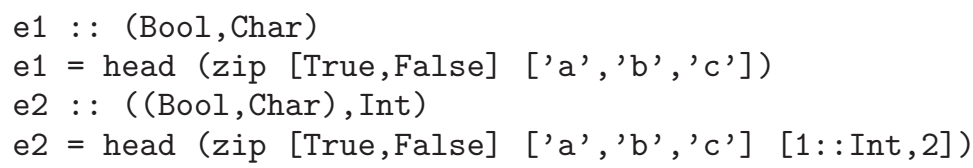

\subsection{Functional Dependencies Are Tricky}

As we have seen, functional dependencies allow the programmer to exert control over the type inference process. However, used uncritically, this additional control can have unexpected consequences. Specifically: they may lead to inconsistency, whereby the type inference engine deduces nonsense such as Int = Bool; and they may lead to non-termination, whereby the type inference engine goes into an infinite loop. We illustrate each of these difficulties with an example.

Example 5. Suppose we add instance Mul Int Float Int to Example 3 That is, we have the following declarations:

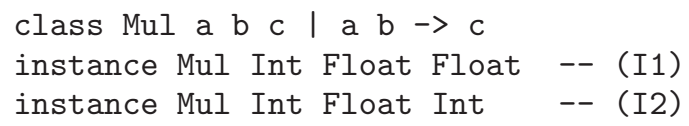

Note that the first two parameters are meant to uniquely determine the third parameter. In case type inference encounters Mul Int Float a we can either argue that $\mathrm{a}=$ Int because of instance declaration (I2). However, declaration (I1) would imply a=Float. These two answers are inconsistent, so allowing both (I1) and (I2) makes the whole program inconsistent, which endangers soundness of type inference. 
Example 6. Assume we add the following function to the classes and instances in Example 3 .

$$
\mathrm{f} \mathrm{b} x \mathrm{y}=\text { if } \mathrm{b} \text { then }(*) \mathrm{x}[\mathrm{y}] \text { else } \mathrm{y}
$$

The program text gives rise to the constraint Mul a (Vec b) b. The improvement rules connected to instance Mul a b c $\Rightarrow$ Mul a (Vec b) (Vec c) imply that $\mathrm{b}=\mathrm{Vec} \mathrm{c}$ for some $\mathrm{c}$; applying this substitution gives the constraint $\mathrm{Mul}$ a $(\operatorname{Vec}(\operatorname{Vec} c))$ (Vec c). But this constraint can be simplified using the instance declaration, giving rise to the simpler constraint Mul a (Vec c) c. Unfortunately, now the entire chain of reasoning simply repeats! We find that type inference becomes suddenly non-terminating. Note that the instances (without the functional dependency) are terminating.

The bottom line is this. We want type inference to be sound and decidable. Functional dependencies threaten this happy situation. The obvious solution is to place restrictions on how functional dependencies are used, so that type inference remains well-behaved, and that is what we discuss next.

\subsection{Jones's Functional Dependency Restrictions}

We assume that $f v(t)$ takes a syntactic term $t$ and returns the set of free variables in $t$. A substitution $\theta=\left[t_{1} / a_{1}, \ldots, t_{n} / a_{n}\right]$ simultaneously replaces each $a_{i}$ by its corresponding $t_{i}$.

In Jones's original paper Jon00, the following restrictions are imposed on functional dependencies.

Definition 1 (Haskell-FD Restrictions). Consider a class declaration

$$
\text { class } C \Rightarrow \mathrm{TC} a_{1} \ldots a_{n} \mid f d_{1}, \ldots, f d_{m}
$$

where the $a_{i}$ are type variables and $C$ is the class context consisting of a (possibly empty) set of type class constraints. Each $f d_{i}$ is a functional dependency of the form $11 a_{i_{1}}, \ldots, a_{i_{k}} \rightarrow a_{i_{0}}$ where $\left\{i_{0}, i_{1}, \ldots, i_{k}\right\} \subseteq\{1 \ldots n\}$. We commonly refer to $a_{i_{1}}, \ldots, a_{i_{k}}$ as the domain and $a_{i_{0}}$ as the range.

The following conditions must hold for functional dependency $f d_{i}$ :

Consistency. Consider every pair of instance declarations

$$
\begin{aligned}
& \text { instance } \ldots \Rightarrow \text { TC } t_{1} \ldots t_{n} \\
& \text { instance } \ldots \Rightarrow \text { TC } s_{1} \ldots s_{n}
\end{aligned}
$$

for a particular type class TC. Then, for any substitution $\phi$ such that

$$
\phi\left(t_{i_{1}}, \ldots, t_{i_{k}}\right)=\phi\left(s_{i_{1}}, \ldots, s_{i_{k}}\right)
$$

we must have that $\phi\left(t_{i_{0}}\right)=\phi\left(s_{i_{0}}\right)$.

\footnotetext{
${ }^{1}$ Haskell systems that allow functional dependencies usually allow dependencies of the form $\mathrm{a} \rightarrow \mathrm{b} \mathrm{c}$, with multiple type variables to the right of the arrow. But this is equivalent to the form $a \rightarrow b, a \rightarrow c$, so in the rest of the paper we only deal with the case where there is a single type variable to the right of the arrow.
} 
Termination. For each instance ... $\Rightarrow \mathrm{TC} t_{1} \ldots t_{n}$ we must have that

$$
f v\left(t_{i_{0}}\right) \subseteq f v\left(t_{i_{1}}, \ldots, t_{i_{k}}\right)
$$

The first of these conditions rules out inconsistent instance declarations (see Example 5); and it turns out that the second ensures termination, although the informal argument in Jones's original paper does not mention termination as an issue. In particular, the second restriction makes illegal the recursive Vec instance in Example 3 (since $f v(c) \nsubseteq f v(a, b)$ ), and hence prevents the divergence of Example6.

To the best of our knowledge, no one has proved that the restrictions given above ensure sound and decidable type inference. We do so, for the first time, in Section 3 .

While these two restrictions make the system well-behaved, it is natural to ask whether either condition could be weakened. The consistency condition seems entirely reasonable, but we have seen many examples in which the termination restriction excludes entirely reasonable and useful programs. Besides Examples 3 (which appears in Jones's original paper) and 4. there are a number of other examples in the literature which break the termination condition Kar03,WW03, CK03. In Section 4.1. we propose a more liberal form of FDs which allows for breaking the termination condition under some additional conditions.

\section{$3 \quad$ Functional Dependencies Expressed Using CHRs}

In this section we explain how to translate functional dependencies into a lowerlevel notation, called Constraint Handling Rules (CHRs) Frü98. This translation has two benefits: it allows us to give a more precise account of exactly what functional dependencies mean; and it allows us to formally verify that Jones's conditions are sufficient to ensure sound and decidable type inference.

Example \%. Let us return to the collection example:

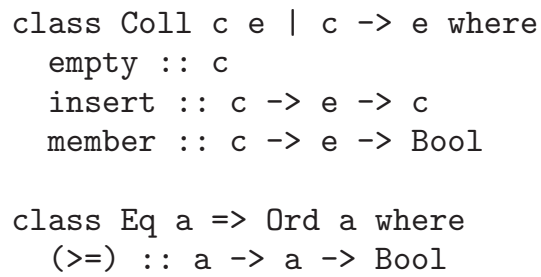

From the functional dependency $\mathrm{c}^{->\mathrm{e}}$ we generate the two improvement rules which we shall express using the following CHRs:

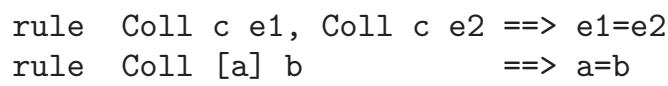

Informally, the first rule says that if the two constraints (Coll c e1) and (Coll c e2) both hold, then it must be that e1 and e2 are the same type. This 
rule is generated from the class declaration alone, and expresses the idea that c uniquely determines e. The second rule is generated from the instance declaration, together with the functional dependency, and states that if (Coll [a] b) holds, then it follows that $\mathrm{a}=\mathrm{b}$. During type inference, the inference engine is required to solve sets of constraints, and it can apply these improvement rules to narrow its choices.

These CHRs have one or more type-class constraints on the left hand side, and one or more equality constraints on the right. The logical interpretation of $==>$ is implication. Its operational interpretation - that is, its effect on the type inference process - is this: when the type inference engine sees constraints matching the left hand side, it adds the constraints found on the right-hand side.

Superclass relations also generate CHR rules. The superclass relationship class $\mathrm{Eq} \mathrm{a} \Rightarrow$ Ord a where... generates the CHR

rule Ord a $==>$ Eq a

Informally, the rule states that if the constraint Ord a holds then also the constraint Eq a holds. During typing this rule is used to check that all superclass constraints are also satisfied.

The instance declaration above also generates the following CHR rule, which allows us to simplify sets of constraints to remove class constraints which are known to hold.

rule Coll [a] a

Informally, the rule states that the constraint Coll [a] a holds if and only if Ord a holds. The logical interpretation of the $\Leftrightarrow=>$ is bi-implication, while the operational interpretation is to replace the constraints on the left hand side by those on the right hand side.

Although not relevant to the content of this paper, the rule generated from the instance is also intimately connected to the evidence translation for the program above, we refer readers to [SS02] for more details.

\subsection{Translation to CHRs}

Formalising the translation given above, class and instance declarations are translated into CHRs as follows:

Definition 2 (CHR Translation). Consider a class declaration

$$
\text { class } C \Rightarrow \mathrm{TC} a_{1} \ldots a_{n} \mid f d_{1}, \ldots, f d_{m}
$$

where the $a_{i}$ are type variables and each functional dependency $f d_{i}$ is of the form $a_{i_{1}}, \ldots, a_{i_{k}} \rightarrow a_{i_{0}}$, where $\left\{i_{0}, i_{1}, \ldots, i_{k}\right\} \subseteq\{1 \ldots n\}$. From the class declaration we generate the following CHRs:

Class CHR: rule TC $a_{1} \ldots a_{n}==C$

Functional dependency CHR: for each functional dependency $f d_{i}$ in the class declaration, we generate

rule TC $a_{1} \ldots a_{n}$, TC $\theta\left(b_{1}\right) \ldots \theta\left(b_{n}\right)=\Rightarrow a_{i_{0}}=b_{i_{0}}$ where $\theta\left(b_{i_{j}}\right)=a_{i_{j}}, j>0$ and $\theta\left(b_{l}\right)=b_{l}$ if $\neg \exists j . l=i_{j}$. 
In addition, for each instance declaration of the form

$$
\text { instance } C \Rightarrow \mathrm{TC} t_{1} \ldots t_{n}
$$

we generate the following CHRs:

Instance CHR: rule TC $t_{1} \ldots t_{n} \Leftrightarrow=$. In case the context $C$ is empty, we introduce the always-satisfiable constraint True on the right-hand side of generated CHRs.

Instance improvement CHR: for each functional dependency $f d_{i}$ in the class declaration, rule TC $\theta\left(b_{1}\right) \ldots \theta\left(b_{n}\right)==>t_{i_{0}}=b_{i_{0}}$ where $\theta\left(b_{i_{j}}\right)=t_{i_{j}}, j>0$ and $\theta\left(b_{l}\right)=b_{l}$ if $\neg \exists j . l=i_{j}$.

If $p$ is a set of class and instance declarations, we define $\operatorname{Simp}(\mathrm{p})$ to be the set of all instance CHRs generated from $p$; and $\operatorname{Prop}(\mathrm{p})$ to be the set of all class, functional-dependency and instance-improvement CHRs generated from $p^{2}$. We define $\operatorname{Prop}_{\text {class }}(\mathrm{p})$ to be the set of all class CHRs in $\operatorname{Prop}(\mathrm{p})$, and similarly $\operatorname{Prop}_{\text {inst }}(\mathrm{p})$ to be the set of all instance improvement CHRs in Prop (p),

The class and instance CHRs, $\operatorname{Prop}_{\text {class }}(p) \cup \operatorname{Simp}(p)$, are standard Haskell, while the functional-dependency and instance-improvement CHRs arise from the functional-dependency extension to Haskell.

For convenience, in the case where the functional dependency $a_{i_{1}}, \ldots, a_{i_{k}} \rightarrow a_{i_{0}}$ imposed on $\mathrm{TC}$ is full, that is, when $k=n-1$, we are able to combine the instance improvement and instance rule into one rule. In such a situation, for each instance $C \Rightarrow \mathrm{TC} t_{1} \ldots t_{n}$ and full functional dependency $a_{i_{1}}, \ldots, a_{i_{k}} \rightarrow a_{i_{0}}$ we generate the following CHR: rule TC $\theta\left(b_{1}\right) \ldots \theta\left(b_{n}\right) \Longleftrightarrow t_{i_{0}}=b_{i_{0}}$, C where $\theta\left(b_{i_{j}}\right)=t_{i_{j}}, j>0$ and $\theta\left(b_{l}\right)=b_{l}$ if $\neg \exists j . l=i_{j}$.

By having a uniform description of (super) class and instance relations and FDs in terms of CHRs, we can establish some important criteria (in terms of CHRs) under which type inference is sound and decidable.

\subsection{Main Result}

The translation to CHRs allows us to phrase the entire type inference process as CHR solving. We know from earlier work that if a set of CHRs is (a) confluent, (b) terminating, and (c) range-restricted (all terms that we explain shortly) we achieve type inference that is sound (all answers are correct), complete (if there is an answer then type inference will provide us with an answer), and decidable (the type inference engine always terminates) [SS02].

Then our main result is as follows:

Theorem 1 (Soundness and Decidability). Let $p$ be a set of Haskell class and instance declarations which satisfies the Haskell-FD restrictions (see Definition (1). Let $\operatorname{Simp}(p)$ and $\operatorname{Prop}(p)$ be the sets of CHRs defined by Definition [2] If the set $\operatorname{Prop}_{\text {class }}(p) \cup \operatorname{Simp}(p)$ of CHRs is confluent, terminating and rangerestricted then $\operatorname{Simp}(p) \cup \operatorname{Prop}(p)$ is confluent, terminating and range-restricted.

2 "Simp" is short for "simplification rule" and "Prop" for "propagation rule", terminology that comes from the CHR literature. 
The design of Haskell 98 ensures that the CHRs $\operatorname{Prop}_{\text {class }}(p) \cup \operatorname{Simp}(p)$, which represent the Haskell type system with no FD extension, are indeed confluent, terminating and range-restricted. Hence, our theorem says that provide the FDs satisfy the Jones restrictions, then type inference is sound and decidable.

To explain this result we need to say what we mean for a set of CHRs to be confluent, terminating, and range restricted.

Confluence. Recall Example 5 whose translation to CHRs is as follows (note that the functional dependency is fully imposed).

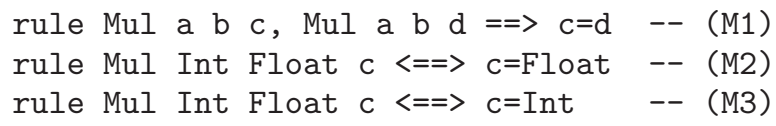

We find two contradicting CHR derivations. We write $C \hookrightarrow_{R} D$ to denote the CHR derivation which applies rule $(\mathrm{R})$ to constraint store $C$ yielding store $D$. E.g. consider Mul Int Float $c \hookrightarrow_{M 2} c=$ Float and Mul Int Float $c \succ_{M 3} c=$ Int. The problem with the code of Example 5 manifests itself in the CHR rules as non-confluence. That is there are two possible sequences of applying rules, that lead to different results. Just considering the rules as logical statements, the entire system is unsatisfiable; that is, there are no models which satisfy the above set of rules.

Non-confluence also arises in case of "overlapping" instances. Assume we add the following declaration to the code of Example 7 .

instance Eq a ==> Coll [a] a where

In case type inference encounters Coll $[t] t$ we can either reduce this constraint to Ord $t$ (by making use of the original instance) or Eq $t$ (by making use of the above instance). However, both derivations are non-joinable. In fact, a common assumption is that instances must be non-overlapping, in which case non-confluence only occurs due to "invalid" FDs.

We note that the consistency condition alone is not sufficient to guarantee confluence (assuming that instances and super classes are already confluent of course).

Example 8. The following code fragment forms part of a type-directed evaluator.

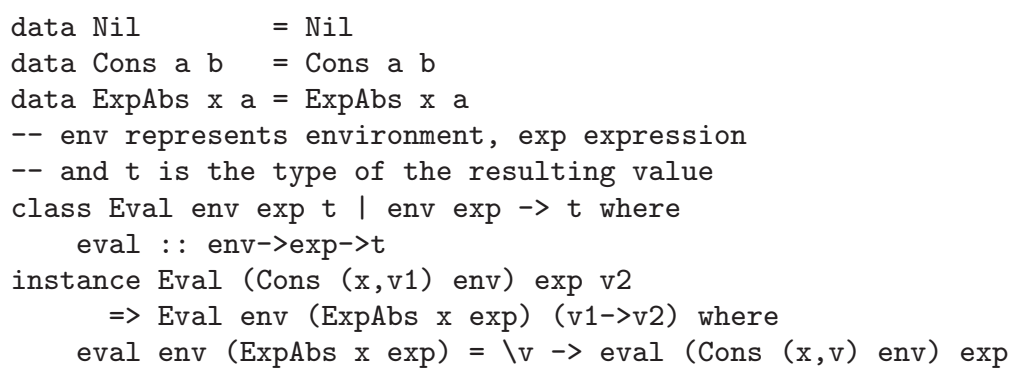

The translation to CHRs yields

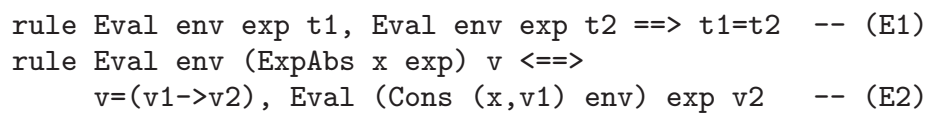


Note that the termination condition is violated but the consistency condition is trivially fulfilled (there is only one instance). However, we find that CHRs are terminating but non-confluent. E.g. we find that (applying (E2) twice)

$$
\begin{gathered}
\text { Eval env }(\text { ExpAbs } x \text { exp }) t_{1}, \text { Eval env }(\text { ExpAbs } x \text { exp }) t_{2} \\
\left.\longmapsto^{*} t_{1}=v_{1} \rightarrow v_{2}, \text { Eval (Cons }\left(x, v_{1}\right) \text { env }\right) \text { exp } v_{2}, \\
t_{2}=v_{3} \rightarrow v_{4}, \text { Eval (Cons }\left(x, v_{3}\right) \text { env) exp } v_{4}
\end{gathered}
$$

Note that rule (E1) cannot be applied on constraints in the final store. But there is also another non-joinable derivation (applying rule (E1) then (E2))

$$
\begin{gathered}
\text { Eval env }\left(\text { ExpAbs x exp) } t _ { 1 } \text { , Eval env } \left(\text { ExpAbs x exp) } t_{2}\right.\right. \\
\longmapsto^{*} t_{1}=t_{2}, t_{1}=v_{5} \rightarrow v_{6} \text {,Eval }\left(\text { Cons }\left(x, v_{5}\right) \text { env }\right) \text { exp } v_{6}
\end{gathered}
$$

So the "termination condition" is perhaps mis-named; in this example, its violation leads to non-confluence rather than non-termination.

Termination. Recall Example 3 The translation to CHRs yields (among others) the following.

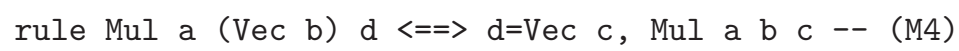

The program text in Example 6 gives rise to Mul a (Vec b) b. We find that

$$
\begin{aligned}
& \quad \text { Mula }(\text { Vec } b) b \\
& \longmapsto_{M 4} \text { Mul } a(\text { Vec } c) c, c=V e c b \\
& \longmapsto_{M 4} \text { Mul } a(V e c d) d, d=V e c c, c=V e c b \\
& \ldots
\end{aligned}
$$

That is, the CHR derivation, and hence type inference, is non-terminating. The important point here is that non-termination was introduced through the FD.

For the purpose of this paper, we generally assume that instance CHRs are terminating. There exists some sufficient criteria to ensure that instance CHRs are terminating, e.g. consider Pey99. Clearly, we can possibly identify further classes of terminating instance CHRs which we plan to pursue in future work. Note that, when a set of CHRs are terminating, we can easily test for confluence by checking that all "critical pairs" are joinable Abd97.

Range restriction. Range-restrictedness is the third condition we impose on CHRs. We say a CHR is range-restricted iff grounding all variables on the lefthand side of a CHR, grounds all variables on the right-hand side.

Example 9. Consider

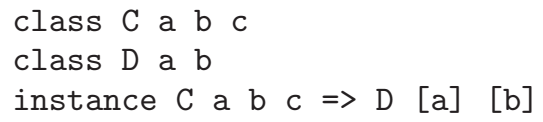

Our translation to CHRs yields

rule D [a] [b] $\Leftrightarrow=>$ C a b c -- (D1) 
Note that rule (D1) is not range-restricted. After grounding the left-hand side, we still find non-ground variable $\mathrm{c}$ on the right-hand side. Range-restrictedness ensures that no unconstrained variables are introduced during a derivation and is a necessary condition for complete type inference. We refer readers to SS02 for more details.

\section{Extensions}

In turn we discuss several extensions and variations of functional dependencies.

\subsection{More Liberal Functional Dependencies}

Earlier in the paper we argued that, while Jones's consistency condition is reasonable, the termination condition is more onerous than necessary, because it excludes reasonable and useful programs (Section 2.2). In this section we suggest replacing the termination restriction with the following weaker one, with the goal of making these useful programs legal.

Definition 3 (Liberal-FD). Consider a class declaration

$$
\text { class } C \Rightarrow \text { TC } a_{1} \ldots a_{n} \mid f d_{1}, \ldots, f d_{m}
$$

where the $a_{i}$ are type variables and $C$ is the class context consisting of a (possibly empty) set of type class constraints. Each $f d_{i}$ is a functional dependency of the form $a_{i_{1}}, \ldots, a_{i_{k}} \rightarrow a_{i_{0}}$ where $\left\{i_{0}, i_{1}, \ldots, i_{k}\right\} \subseteq\{1 \ldots n\}$.

In addition to the consistency condition (see Definition 1 ), the following condition must hold for more liberal functional dependency $f d_{i}$ :

Context Consistency. For each instance $C \Rightarrow \mathrm{TC} t_{1} \ldots t_{n}$ we must have that $f v\left(t_{i 0}\right) \subseteq \operatorname{closure}\left(C, f v\left(t_{i 1}, \ldots, t_{i k}\right)\right)$ where

$$
\begin{gathered}
\operatorname{closure}(C, v s)=\bigcup_{\mathrm{TC}} t_{1} \ldots t_{n} \in C\left\{f v\left(t_{i_{0}}\right) \mid f v\left(t_{i_{1}}, \ldots, t_{i_{k}}\right) \subseteq v s\right\} \\
\quad \mathrm{TC} a_{1} \ldots a_{n} \mid a_{i_{1}}, \ldots, a_{i_{k}} \rightarrow a_{i_{0}}
\end{gathered}
$$

The basic idea of the context consistency condition is that the variables in the range are captured by some FDs imposed on type classes present in the context. Note that although the context consistency condition resembles a more "liberal" version of the termination condition, context consistency does not prevent non-termination. Example 3 satisfies both of the above conditions, however, resulting CHRs are non-terminating. More precisely, adding the improvement rules $\operatorname{Prop}(p)$ to a terminating set $\operatorname{Simp}(p)$ of instance CHRs yields a non-terminating set $\operatorname{Simp}(p) \cup \operatorname{Prop}(p)$. Hence, for the following result to hold we need to assume that CHRs are terminating.

Theorem 2 (More Liberal FDs Soundness). Let $p$ be a set of Haskell class and instance declarations which satisfies the Liberal-FD restrictions. Let $\operatorname{Simp}(p)$ and $\operatorname{Prop}(p)$ be defined by Definition Q If the set $\operatorname{Simp}(p) \cup \operatorname{Prop}(p)_{\text {class }}$ $i s$ confluent and range-restricted and $\operatorname{Simp}(p) \cup \operatorname{Prop}(p)$ is terminating, then $\operatorname{Simp}(p) \cup \operatorname{Prop}(p)$ is confluent and range-restricted. 
Note that Example 4 also satisfies the more liberal FD conditions. According to Definition 2 we generate the following improvement rules. Note that the functional dependency imposed is full. For simplicity, we only focus on improvement rules.

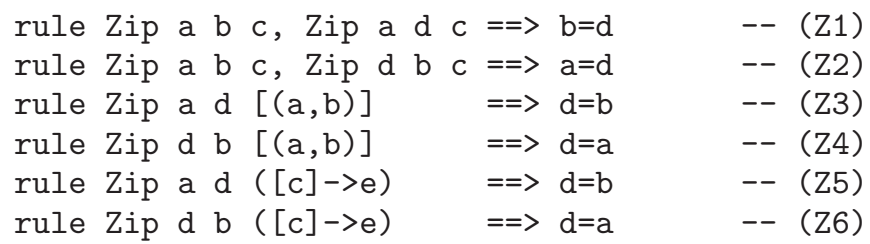

Rules (Z5) and (Z6) are generated from the second instance. Note that both rules introduce some new variables since we violate the termination condition. However, both rules are harmless. Effectively, we can replace them by

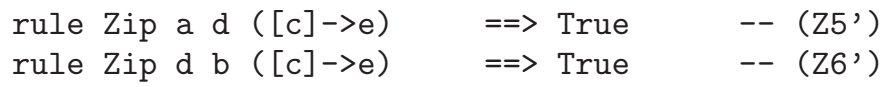

which makes them trivial. Hence, we can omit them altogether. We observe that we can "safely" violate the termination condition (without breaking termination) in case the improvement rules generated are trivial, i.e. the right-hand side of CHRs can be replaced by the always true constraint. This is always the case if the range component of an instance is a variable.

\subsection{Stronger Improvement}

There are situations where FDs do not enforce sufficient improvement. Note that the inferred types of e1 and e2 in Example 4 are

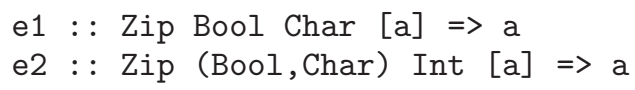

rather than

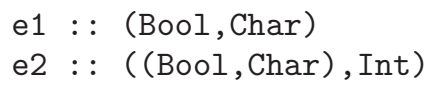

For example rule (Z3) states that only if we see Zip a d $[(a, b)]$ we can improve $\mathrm{d}$ by $\mathrm{b}$. However, in case of e1 we see Zip Bool Char [a], and we would like to improve a to (Bool, Char). Indeed, in this context it is "safe" to replace rules (Z3) and (Z4) by

rule Zip a b $[\mathrm{c}]==>\mathrm{c}=(\mathrm{a}, \mathrm{b}) \quad--(\mathrm{Z} 34)$

which imposes stronger improvement to achieve the desired typing of e1 and e2. Note that rule (Z34) respects the consistency and termination conditions (assuming we enforce these conditions for user-provided improvement rules). Hence, we retain confluence and termination of CHRs.

Of course, if a user-provided improvement violates any of the sufficient conditions, it is the user's responsibility to ensure that resulting CHRs are confluent and terminating. 


\subsection{Instance Improvement Only}

Instead of stronger improvement it might sometimes be desirable to omit certain improvement rules. For example, in case the context consistency condition is violated, we can recover confluence by dropping the functional dependency rule.

Theorem 3 (Instance Improvement Soundness). Let $p$ be a set of Haskell class and instance declarations which satisfies the Haskell-FD consistency restriction. If the set $\operatorname{Simp}(p) \cup \operatorname{Prop}_{\text {class }}(p)$ is confluent and range-restricted and $\operatorname{Simp} \cup \operatorname{Prop}_{\text {class }}(p) \cup \operatorname{Prop}_{\text {inst }}(p)$ is terminating, then $\operatorname{Sim} p(p) \cup \operatorname{Prop}_{\text {class }}(p) \cup$ Prop $_{\text {inst }}(p)$ is confluent and range-restricted.

Here is a (confluent) variation of Example 8 where we only impose the instance improvement rule.

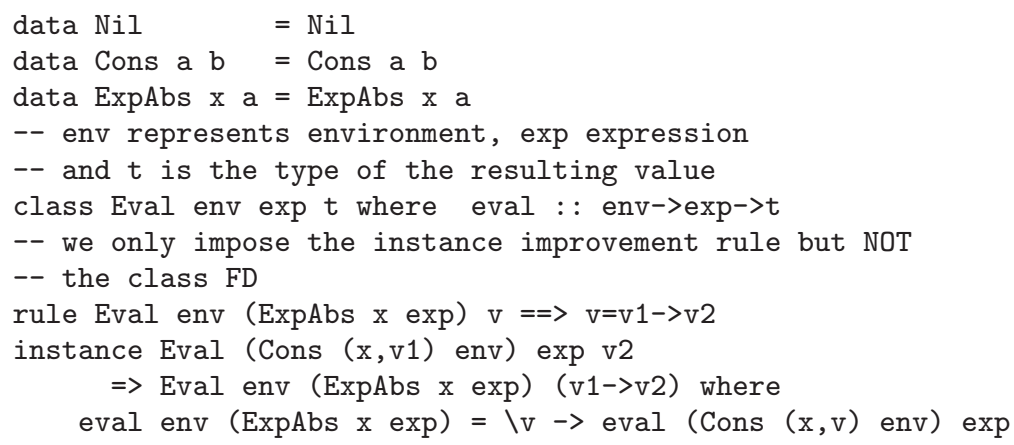

\section{$5 \quad$ Related Work}

The idea of improving types in the context of Haskell type classes is not new. For example, Chen, Hudak and Odersky [CHO92] introduce type classes which can be parameterized by a specific parameter. For example, the declaration class SM m r | m->r from Example2 2 can be expressed as the parametric declaration class m: :SM $r$. Interestingly, they impose conditions similar to Jones's consistency and termination condition to achieve sound and decidable type inference. However, their approach is more limited than ours. Functional dependencies must be always of the form $a->b$ where $b$ is not allow to appear in the domain of any other functional dependency. Furthermore, they do not consider any extensions such as more liberal FDs.

In [Jon95], Jones introduces a general theory of simplifying and improving types as a refinement of his theory of qualified types Jon92. However, he does not provide any formal results which improvement strategies lead to sound and decidable type inference.

Subsequently, Jones extends multi-parameter type classes with functional dependencies [Jon00]. He states some conditions (consistency and termination) which in this paper we finally verify as sufficient to ensure sound and decidable type inference. Surprisingly, he introduces Example 3 (which breaks the termination condition) as a motivation for functional dependencies. 
Duggan and Ophel DO02 describe an improvement strategy, domain-driven unifying overload resolution, which is very similar to functional dependencies. Indeed, they were the first to point out the potential problem of non-termination of type inference. However, they do not discuss any extensions such as more liberal FDs nor do they consider how to cope with the termination problem.

Stuckey and Sulzmann SS02] introduce a general CHR-based formulation for type classes. They establish some general conditions, e.g. termination and confluence, in terms of CHRs under which type inference is sound and decidable. Here, we rephrase functional dependencies as a particular instance of their framework.

\section{Conclusion}

We have given a new perspective on functional dependencies by expressing the improvement rules implied by FDs in terms of CHRs. We have verified, for the first time, that the conditions (termination and consistency, see Definition 1) stated by Jones are sufficient to guarantee sound and decidable type inference (see Theorem 1).

There are many examples which demand dropping the termination condition. For this purpose, we have introduced more liberal FDs in Section 4.1. We have identified an additional condition (context consistency) which guarantees confluence (see Theorem 2). We have also discussed further useful extensions such as stronger improvement rules (Section 4.2) and instance improvement rules only (Section 4.3).

For such extensions it becomes much harder to guarantee decidability (unless the generated improvement rules are trivial). For example, the more liberal FD conditions only ensure soundness but not decidability. We are already working on identifying further decidable classes of CHRs. We expect to report results on this topic in the near future.

In another line of future work we plan to investigate how to safely drop the consistency condition. Consider

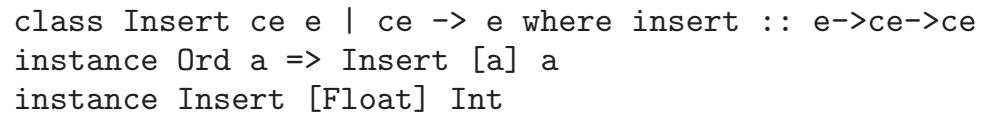

Our intention is to insert elements into a collection. The class declaration states that the collection type uniquely determines the element type. The first instance states that we can insert elements into a list if the list elements enjoy an ordering relation. The second instance states that we have a special treatment in case we insert Ints into a list of Floats (for example, we assume that Ints are internally represented by Floats). This sounds reasonable, however, the above program is rejected because the consistency condition is violated. To establish confluence we seem to require a more complicated set of improvement rules. We plan to pursue this topic in future work.

Acknowledgements. We thank Jeremy Wazny and the reviewers for their comments. 


\section{References}

[Abd97] S. Abdennadher. Operational semantics and confluence of constraint propagation rules. In Proc. of CP'97, volume 1330 of LNCS, pages 252-266. Springer-Verlag, 1997.

[CHO92] K. Chen, P. Hudak, and M. Odersky. Parametric type classes. In Proc. of ACM Conference on Lisp and Functional Programming, pages 170-191. ACM Press, June 1992.

[CK03] M. Chakravarty and S. Keller. Classy type analysis, 2003.

[DO02] D. Duggan and J. Ophel. Type-checking multi-parameter type classes. Journal of Functional Programming, 12(2):133-158, 2002.

[DPJSS03] G. J. Duck, S. Peyton-Jones, P. J. Stuckey, and M. Sulzmann. Sound and decidable type inference for functional dependencies. Technical report, National University of Singapore, 2003.

http://www . comp.nus.edu.sg/ sulzmann/chr/download/fd-chr.ps.gz.

[Frü95] T. Frühwirth. Constraint handling rules. In Constraint Programming: Basics and Trends, volume 910 of LNCS. Springer-Verlag, 1995.

[Frü98] T. Frühwirth. Theory and practice of constraint handling rules. Journal of Logic Programming, 37(1-3):95-138, 1998.

[Jon92] M. P. Jones. Qualified Types: Theory and Practice. PhD thesis, Oxford University, September 1992.

[Jon95] M. P. Jones. Simplifying and improving qualified types. In FPCA '95: Conference on Functional Programming Languages and Computer Architecture. ACM Press, 1995.

[Jon00] M. P. Jones. Type classes with functional dependencies. In Proc. of the 9th European Symposium on Programming Languages and Systems, ESOP 2000, volume 1782 of LNCS. Springer-Verlag, March 2000.

[Kar03] J. Karczmarczuk. Structure and interpretation of quantum mechanics a functional framework. In Proc. of Haskell Workshop'03, pages 50-61. ACM Press, 2003.

[Pey99] S. Peyton Jones et al. Report on the programming language Haskell 98, February 1999. http://haskell.org.

[SS02] P. J. Stuckey and M. Sulzmann. A theory of overloading. In Proc. of ICFP'02, pages 167-178. ACM Press, 2002.

[WW03] G. Washburn and S. Weirich. Boxes go bananas: encoding higher-order abstract syntax with parametric polymorphism. In Proc. of ICFP'03, pages 249 - 262. ACM Press, 2003. 